\title{
Quiénes son los visitantes de los museos de arte.Particularidades de los públicos del fin de semana en el Museo de Arte de Tigre (Argentina)
}

Who are the visitors of the art museums.

Particularities of the publics of the weekends at the Art Museum of Tigre (Argentina)

https://doi.org/10.1590/1982-02672020v28e1 1

\section{ALEJANDRA GABRIELA PANOZZO ZENERE'}

https://orcid.org/0000-0003-1929-0434

Universidad Nacional de Rosario / Rosario, Argentina.

RESUMEN: Saber quiénes son sus visitantes es una de las principales tareas de los museos en la actualidad, en la medida en que fluctúan entre ajustarse a las imposiciones del sistema capitalista y convertirse en instituciones democráticas y participativas. No obstante, este aspecto resulta aun más dificultoso en los museos de arte, en virtud de los atributos particulares de su estructura y actores. El presente trabajo tiene como objetivo aproximar algunos criterios que permitan conocer quiénes son los visitantes de los museos de arte creados en Argentina desde el inicio del nuevo milenio. Dada la magnitud del desafío, se procura, con el foco puesto en un caso particular, trazar algunos lineamientos sostenidos en los enfoques teórico-críticos aplicados a la museología y en los estudios de públicos. En cuanto a lo metodológico, se recurrió a un estudio de visitantes que apeló a orientaciones cuantitativas y cualitativas, realizado en el Museo de Arte de Tigre durante 2017, centrado en los públicos del fin de semana. Se considera que, al indagar los rasgos singulares de esta entidad patrimonial, pueden identificarse algunos indicios que permitan comenzar a trazar un perfil, tanto general como específico, de los visitantes de los museos de arte.

PALABRAS CLAVE: Visitantes. Micropúblicos. Preferencias. Museos de arte.

\begin{abstract}
1. Doctorado en Comunicación Social por la Universidad Nacional de Rosario (UNR). Magister en Industrias Culturales por la Universidad Nacional de Quilmes (UNQ). Licenciada y Profesora en Bellas Artes con especialidad en Teoría y Crítica (UNR). E-mail: <panozzozenere. alejandra@gmail.com>.
\end{abstract}


ABSTRACT: Knowing who its visitors are is one of the main tasks of museums today, as they fluctuate between conforming to the impositions of the capitalist system and becoming democratic and participatory institutions. However, this aspect is even more difficult in the arts museums because of the particular attributes of their structure and its diverse actors. The present work has as main object to bring near some criteria that may allow to know who are the visitors of the art museums in Argentina since the beginning of the new millennium. Given the extent of the challenge, we'll try, focusing in a particular case, draw some guidelines sustained in the theorical-critical approaches applied to museology and studies of the publics. In terms of methodology, a visitor study was carried out at the Tigre Art Museum in 2017, using both quantitative and qualitative guidelines, focusing on weekend audiences. It is considered that, when we question the singularities of this entity, there are some indications that a general and specific profile of visitors to art museums can begin to be identified.

KEYWORDS: Visitors. Micropublics. Preferences. Art museums.

RESUMO: Saber quem são seus visitantes é uma das principais tarefas dos museus na atualidade, na medida em que flutuam entre amoldar-se às imposições do sistema capitalista e converter-se em instituições democráticas e participativas. Não obstante, este aspecto resulta ainda mais dificultoso nos museus de arte, devido aos atributos particulares de sua estrutura e atores. $\bigcirc$ presente trabalho tem por objetivo aproximar alguns critérios que permitam conhecer quem são os visitantes dos museus de arte criados na Argentina desde o início do novo milênio. Dada a magnitude do desafio, e com o foco posto em um caso particular, buscou-se traçar alguns lineamentos sustentados nos enfoques teórico-críticos aplicados à museologia e nos estudos de públicos. Quanto à metodologia, a pesquisa teve como base um estudo de visitantes que apelou a orientações quantitativas e qualitativas, realizado no Museu de Arte de Tigre durante $\mathrm{o}$ ano de 2017, focado nos públicos de fim de semana. Considera-se que, ao indagarmos os traços singulares desta entidade, podem ser identificados alguns indícios que permitam começar a esboçar um perfil, tanto geral quanto específico, dos visitantes dos museus de arte.

Palavras-chave: Visitantes. Micropúblicos. Preferências. Museus de arte. 


\section{INTRODUCCIÓN}

Este trabajo intenta reflexionar acerca de una serie de resultados iniciales, obtenidos tras la puesta en marcha de un proyecto de investigación posdoctoral (2017-20 19) cuyo propósito es indagar la experiencia de la visita a los museos de arte argentinos creados a partir del siglo XXI. El objetivo de dicha investigación consiste en reconocer aspectos que favorezcan el acercamiento entre estos dos actores - los museos y sus visitantes-, sin perder de vista las lógicas que caracterizan a las sedes museales artísticas en la contemporaneidad.

Nuestra búsqueda se abre con una pregunta: ¿̇quiénes visitan los museos de arte argentinos creados en el inicio del nuevo milenio? El primer museo en que pusimos a prueba esta interrogación es el Museo de Arte de Tigre; una institución joven - que data de 2006 - situada por fuera del circuito porteño - cabe recordar que la centralidad de la capital argentina, Buenos Aires, definió tanto a las instituciones culturales del país como a su sistema artístico-. En sus instalaciones realizamos, durante el año 2017, un primer estudio cuantitativo-cualitativo de visitantes que desde cualidades específicas y una realidad particular, posibilita, por un lado, reconocer diferentes perfiles y tipologías de visitantes de este tipo de establecimiento; mientras que, por otro, arroja luz sobre el conocimiento general de los visitantes de museos de arte argentinos. Encarar un estudio de estas características comienza a ser central para pensar la lógica de las sedes museales en la actualidad y generar, asimismo, una orientación más clara por parte de los museos en la tarea de definir su rol social.

El cruce de los datos obtenidos con una base teórica interdisciplinaria puede contribuir al conocimiento empírico, actual y futuro, de las personas que visitan o podrían visitar los museos de arte. Es de esperar que dicho conocimiento, por otra parte, resulte de gran utilidad para potenciar políticas de gestión orientadas a los distintos públicos que visitan, en el siglo XXl, este tipo de entidad patrimonial en general, y a las de arte en particular. A su vez, tales indagaciones abren un ámbito de reflexión valioso sobre los impulsos contrapuestos - democratizar el acceso a la cultura o mercantilizarloque animan a los museos a actuar bajo las lógicas actuales del sistema capitalista.

\section{LAS DISTINTAS MIRADAS SOBRE LOS VISITANTES DE LOS MUSEOS DE ARTE}

En sus comienzos, los museos se establecieron como una institución cultural centrada en los objetos; y aunque muchos de ellos pretendieran, dada su condición 
2. Pérez Castellano (2017, p. 124)

3. La diferencia que presentan los objetos museales de los museos de arte reside en la categoría que adquieren al ser considerados obras de arte. Esta concepción destaca el valor simbólico por sobre el valor de uso en pos de establecer su condición estética (Mairesse, 2013).

4. Cf. Guasch (2008).

5. Esta es una de las líneas de investigación propia del área de conocimiento museístico conocida como museología crítica (Lorente Lorente, 2003). Sin embargo, preferimos llamarlos enfoques críticos aplicados a la museología, ya que algunos de sus autores trabajan los museos como casos de estudio dentro de un sistema mayor que responde a las disciplinas o campos de investigación en que se encuentran insertos. Para una mayor profundización sobre el tratamiento de esta perspectiva sobre aspectos socioeconómicos y mediáticos revisar Tony Bennett (1996; 2007) y Daniel Sherman y Irit Rogoff (2003).

6. Cf. Krauss (1993).

7. Cf. Laseca (2016).

8. En esta línea, podemos referenciar los análisis de Guido Ballo (1966), Andrea Giunta (2004) y Maria Luz Ruiz Bañón (2017) entre otros.

9. Coelho (2000, p. 420). pública, que sus puertas estaban abiertas para todos los ciudadanos, este propósito quedó relegado a una vaga aspiración, debido a "restricciones implícitas o explícitas que excluían a gran parte de los visitantes y de los públicos potenciales". ${ }^{2}$ En las últimas décadas, ha primado, en general, la idea de volver a dar a estas instituciones una orientación más plural a fin de transformarlas en entidades patrimoniales verdaderamente democráticas; principalmente, se busca atender una mayor diversidad de necesidades e intereses de acuerdo a perfiles más específicos de los públicos.

En los museos de arte, el desplazamiento del centro de atención desde el objeto museístico a los visitantes plantea una serie de problemas cuya complejidad no debe pasarse por alto. En tal sentido, conviene recordar que las cualidades diferenciadoras que poseen sus colecciones ${ }^{3}$ contribuyeron a dar a estos establecimientos una marcada influencia dentro del sistema del arte. ${ }^{4} \mathrm{El}$ constante juego de fuerzas entre sus agentes - artistas, instituciones, teóricos, curadores, públicos, mercado- que caracteriza a dicho sistema artístico constituye, asimismo, una variable que interviene activamente en la vida interna de la sede museal. En este sentido, el museo de arte responde a especificidades de lo artístico que abarcan una amplia gama de lecturas, por ejemplo, desde la Historia del Arte, los Estudios Visuales y los Curatoriales. Está claro, además, que, en el escenario global actual, este establecimiento no puede pensarse como un fenómeno autónomo e independiente, sino que se encuentra atravesado por aspectos socioeconómicos y mediáticos - estos vínculos han sido abordados, especialmente, por los enfoques críticos aplicados a la museología $-{ }^{5}$

Al respecto, consideramos que la afirmación de una estrecha relación entre el museo de arte y el capitalismo tardío, 'bue central para leer el lugar que ocupaba esta institución cultural a fines del siglo XX. Recientemente, se retomaron algunos de estos postulados para afirmar su impulso imparable, ${ }^{7}$ traducida, en el siglo XXI, en una especie de empresa cultural capaz de obtener beneficios de sus productos dirigidos a visitantes específicos. Tanto en uno como en otro momento, el protagonismo otorgado a los públicos ha tenido un peso fundamental; en virtud de ello, las sedes museales artísticas han buscado convertirse en lugares acogedores que ofrecen diversas propuestas, actividades y espacios-servicios con el fin de atender a un mayor número de visitantes.

Pero ¿̇qué pasa en este contexto con los postulados que solían sostener los museos de arte? Por empezar, se abandonó la idea de la existencia de un solo público, en su lugar, comenzaron a explorarse una variedad de registros espectatoriales e imaginarios sobre lo artístico ${ }^{8}$ que permitieron construir un nuevo precepto: "no existe un público de arte sino públicos de arte". ${ }^{9}$ A ello se sumó, particularmente en las últimas décadas del siglo XX, el interés por abordar la visita a los museos en relación con los 
llamados fenómenos de masas. ${ }^{10} \mathrm{~A}$ la luz de estas perspectivas, se hizo visible que los visitantes " ya no solo se veían atraídos por el arte, sino que también operaba en ellos la lógica que mostraba a estas sedes como espacios para la recreación y el ocio.

No obstante, en los últimos años, la idea de multitud - los públicos de los museos abordados en su aspecto macro- se sostiene en la de micropúblicos; ambas nociones se gestaron a partir de los estudios del marketing. ${ }^{12}$ La noción de micropúblico se enfoca en los visitantes en relación con un nicho; se trata de una perspectiva derivada de la teoría Long Tail. ${ }^{13}$ Este tipo de análisis busca conocer las necesidades, los estilos de vida y las motivaciones de los actores de un espacio determinado; y apela, para ello, a la segmentación desde la diferencia y a la singularización como las estrategias más acertadas. Las condiciones que demanda el actual sistema capitalista implican la correspondencia entre una multiplicidad de singularidades para conformar una amplia multitud. En esta línea, se vuelve valioso el uso de los estudios de visitantes o públicos. ${ }^{14}$

A partir de tales indagaciones, los museos pueden conocer, rearticular y proyectarse junto con los visitantes. Las exploraciones, en general, presentan rasgos de tipo sociológico, antropológico y estadístico. Se posibilita, por ejemplo, medir características simples y clásicas, tales como el perfil sociodemográfico, que se basa en rasgos sociales y económicos de los visitantes; aunque, en los últimos años, se reconoce un mayor análisis de la segmentación por edades. ${ }^{15}$ Asimismo, se observa a los visitantes por la presencia en el establecimiento, que permite dividirlos en visitantes frecuentes, participantes ocasionales y no-participantes, ${ }^{16} \mathrm{O}$ en público, público potencial o no público; ${ }^{17}$ o bien, por los contextos en que se encuentran - a saber, escolares, familiares o con necesidades educativas especiales -.$^{18}$

También se detectan, no obstante, análisis más específicos. Por ejemplo, desde los estudios pedagógicos y didácticos, se ha indagado en el aprendizaje o en el modo de procesar la información; ello ha facilitado establecer estilos de aprendizaje que difieren según diversos modos de apropiación: activo -actúa, toca, acciona, resuelve-, reflexivo - observa, escucha, analiza, busca causas y razones-, teórico - organiza lógicamente, deduce, sintetiza, descubre - y pragmático - experimenta, aplica, resuelve- ${ }^{19}$ Otro análisis es aportado por los estudios en recepción, que trabajan el comportamiento en el espacio expositivo; en virtud de lo observado durante el recorrido de una muestra, se formulan una serie de variables etnográficas en las que reconoce bajo el nombre de hormiga a los visitantes más ordenados y disciplinados; bajo el de mariposa, a los que visitan la exposición a su aire, sin lógica ordenada; con el de saltamontes, a quienes disponen de poco tiempo y van dando saltos, visitando solo las obras principales; $y$, finalmente, con el de pez, a quienes se mueven desconfiados a lo largo y ancho del espacio expositivo. ${ }^{20}$
10. Productor, entre otros motivos, de las prácticas que promulgaba el Museo Pompidou (Francia), a finales de la década del setenta.

11. Son identificados bajo la categoría de turista cultural (Olivera, 2008), o visitantes por ocio (Bitgood, 2002).

12. Los artículos dirigidos a micropúblicos comenzó a practicarse, principalmente, en el rubro de los libros y de la gastronomía.

13. Cf. Anderson (2009).

14. "Los estudios de visitantes o estudios de públicos suelen ser trabajados indistintamente en la bibliografía hispano-hablante. Por el contrario, en los textos de habla inglesa se dividen bajo las terminologías visitor studies o visitor research, que suelen relacionarse con el término audience development" (Pérez Castellano, 2016, p. 23).

15. Trabajos que han abordado esta segmentación son de autores como María Shoemaker (1992) y Barry Lord, Dexter Lord y Linda Martin (2012).

16. Cf. Hood (1983).

17. Cf. Sepúlveda Köptcke (2012).

18. Autores que han trabajado este abordaje son Barbara Buttler y Marvin Sussman (1989) y Silvia Alderoqui y Constanza Pedersoli (2011).

19. Cf. Honey; Mumford (1995).

20. Cf. Verón; Levasseur (1984). 
21. Cf. Gardner (1983)

22. Cf. Morris Hargreaves McIntyre (2005)

23. Cf. Orr (2004).

24. Esta segmentación ha sido profundizada en distintos textos de John Falk (2009) y John Falk and Lynn Dierking (2013).

25. Cf. Pekarik, Schreiber, Hanemann, Richmond y Mogel (2014).
Por último, queremos detenernos en los estudios que se desprenden de líneas de investigación que incorporan un enfoque psicológico. Entre ellos, se cuentan, para empezar, los que apelan a las inteligencias o capacidades por medio del aprendizaje; con un marco que deriva de la Teoría de las Inteligencias Múltiples, se explica la manera en que las personas desarrollan distintas capacidades para resolver problemas y aprender del entorno. En esta línea, se brindan clasificaciones según las capacidades: corporal cinestética, visual-espacial, lingüístico-verbal, lógico-matemática, interpersonal, intrapersonal, musical, naturalista y exitista. ${ }^{21}$ También se registran indagaciones que buscan reflexionar sobre el contexto personal y las motivaciones de la visita, o sobre la construcción de significados alrededor de los objetos expuestos; 22 los visitantes son clasificados allí en curiosos, los que deambulan hasta que encuentran algo que llama su atención y atienden a las explicaciones para darle sentido; seguidores, pues seleccionan los objetos a partir de narrativas temáticas; buscadores, ya que cuentan con un entendimiento general del tema y consumen toda la información que presenta la exposición; e investigadores, dado que se trata de visitantes expertos o especializados que seleccionan los objetos según sus conocimientos. Otra perspectiva a considerar se detiene en el comportamiento en relación con la información; ${ }^{23}$ la tipología construida divide a los individuos en consumidores - están motivados por la información y adquieren recuerdos de la experiencia y la recomiendan-; aprendices - buscan información para enfatizar lo que saben y lo vinculan con la experiencia e interactúan con el contenido-; y celebrantes - buscan información en un formato de entretenimiento; son atraídos por los objetos y las narrativas, y establecen vínculos con otros-.

Cabe destacar, finalmente, dos últimos enfoques ubicados en la experiencia. El primero se concentra en búsquedas en torno a la identidad y la experiencia de la visita; ${ }^{24}$ se proponen siete identidades -exploradores, facilitadores, profesionales, contempladores, peregrinos, buscadores de afinidades y buscadores de experienciasque representan los objetivos personales que motivan la visita y en los que se basa la experiencia y la construcción de sentido en el recorrido del museo. El segundo aborda las preferencias durante la experiencia; ${ }^{25}$ entre ellas, se distinguen preferencias por las ideas -interés por los hechos, conceptos, abstracciones, razones, pensamientos-; preferencias por las personas - interés por las historias, conexiones emocionales e interacción social-; preferencia por los objetos -interés y apreciación por los objetos; estética, procesos de elaboración de lenguajes visuales-; y preferencia física -interés por las experiencias sensoriales y el movimiento-.

El panorama ofrecido aquí constituye un escueto recuento, propuesto solo con el fin de exponer parte del amplio abanico de posibilidades existentes en la manera de identificar diferentes perfiles y tipologías de los públicos de los museos. En otras palabras, cualquiera sea la opción que se adopte, constituirá 
un puntapié para comenzar a desentrañar quiénes son los individuos que visitan los museos de arte del siglo XXI y a reconocer la manera en que esta entidad patrimonial instrumenta su rol en la contemporaneidad.

\section{EXPLORACIONES EN TORNO A LOS VISITANTES DE LOS MUSEOS DE ARTE ARGENTINOS}

En el territorio argentino existen, según el último relevamiento realizado por la Guía Nacional de Museos del Ministerio de la Nación en el año 2013, aproximadamente, un total de ciento ocho museos de arte. Todos ellos fueron creados sobre el eje del objeto museístico - la obra de arte-, con lo cual las particularidades de sus colecciones propiciaron la creación de una variedad de sedes museales a lo largo del país. Sin embargo, en lo que va del siglo, se dio un fenómeno alentado por la realidad internacional y el propio contexto argentino que originó un conjunto singular de entidades patrimoniales en todo el territorio - aunque con procesos diversos y temporalidades distintas - ${ }^{26}$ Este fenómeno tuvo lugar bajo las condiciones proyectadas por el sistema artístico y el sistema capitalista en su etapa llamada de globalización neoliberal. ${ }^{27}$ Un rasgo que identificaría a este proceso es el cambio de la relación entre los museos y la ciudad: "la aspiración de toda ciudad ahora no es tener un museo, sino tener un museo anónimo que sea garante de la visibilidad pública de su inscripción en el circuito de ciudades genéricas". ${ }^{28}$

Esta transformación obedece a presiones externas e internas que, en Argentina, comenzaron a configurarse a partir de la década del noventa con la implementación de la reforma neoliberal. ${ }^{29}$ Las presiones externas estuvieron vinculadas, principalmente, con los cambios en los esquemas de financiamiento de los museos. En aquellos años, la gran mayoría de las sedes museales argentinas se sostenían con fondos estatales; pero luego de los sucesos acontecidos a principio del XXI, ${ }^{30}$ tuvieron que generar sus propios recursos $y$ buscar nuevas estrategias. A raíz de ello, se impulsó una ampliación y multiplicación de la escena cultural; se delineó un mapa de incipientes actividades multidisciplinares; se crearon nuevos espacios y se reacondicionaron otros; y se impulsaron eventos masivos. Esta lógica, asociada a la de las industrias del entretenimiento, el ocio y el turismo, encontró una órbita privilegiada de acción en los museos de arte. ${ }^{31}$ Las presiones internas, en cambio, tuvieron lugar a partir de la profesionalización de las áreas de trabajo; aunque recién en estos últimos años se comenzó a generar una práctica museológica más reflexiva y autocrítica, orientada al enfoque de los visitantes.
26. En sintonía con esta lógica, durante la primera década del siglo XXI, se generaron un gran número de museos de Arte. Se generaron, a lo largo del país, establecimientos creados específicamente para albergar y exponer producción contemporánea: el MALBA (Buenos Aires, 2001); el MAC (Salta, 2004); el MAC (Bahía Blanca, 2004); el MACRo (Rosario, 2004); el MACBA (Buenos Aires, 2012); el MAR (Mar del Plata, 2013); entre otros. Asimismo, se generan otros con obras clásicas y modernas, tales como por ejemplo, el MAT (Tigre, 2006) y el Museo Evita-Palacio Ferreyra (Córdoba, 2007). También, podemos nombrar museos de colecciones privadas de artistas como: el Museo de Artes Visuales (Mechita, 2006) generado a partir de la obra de Juan Doffo y el Museo de Arte Contemporáneo creado con la obra de Raúl Lozza (Alberdi, 2003) Por último, no queremos dejar de mencionar los museos de carácter universitario, entre ellos, el Museo de Arte Contemporáneo de la Universidad Nacional del Litoral (Santa Fe, 2000), el Museo de Artes Visuales de la Universidad de Tres de Febrero (Buenos Aires, 2002) y el Museo Internacional Contemporáneo de la Facultad de Arte y Diseño de la Universidad Nacional de Misiones (Oberá, 2015).

27. Para una mayor profundización revisar los textos de Tony Bennett (2007) y María Cristina Chiriguini (2008).

28. Laseca (2016, p. 35).

29. El proceso de reforma neoliberal que afectó a todo el país y que fue llevado adelante por el gobierno menemista (19891995/1995-1999) dio lugar a medidas de apertura y dominio de mercado - liberación financiera, industrial y comercial-que implicaron la 
desarticulación del poder del Estado y la pérdida de la soberanía, junto a la informalización del empleo, el aumento de la desocupación y el abandono de las políticas sociales; todo ello produjo una acelerada marginalidad y empobrecimiento de la población. En consecuencia, luego del gobierno de la Alianza (1999-2001), esta situación no se pudo revertir; ello condujo a una serie de hechos desafortunados.

30. Luego del gobierno de la Alianza (1999-2001), esta situación no se pudo revertir; ello condujo a una serie de hechos desafortunados, que se desarrollaron principalmente durante el 19 y 20 de diciembre de 2001; una seguidilla de saqueos y la insostenible crisis política y social llevaron a las multitudes a ocupar las calles y a exigir la caída del gobierno, que respondió con una brutal represión. A partir de allí se sucederán varios presidentes, hasta el llamado a elecciones que darán origen a un cambio en las políticas implementadas durante esos años.

31. Cf. Panozzo Zenere (2018).

32. Este trabajo comenzó durante la gestión de Ana María Cousillas en conjunto con Mirta Bialogorky, y que continúo, más tarde, con Paola Fritz.

33. Una visión que se vincula con el mensaje criollista que había adoptado la institución a partir de 1955 y que se mantuvo con variantes hasta los años ochenta; fuertemente fijada en el imaginario porteño. Para una mayor profundización revisar los trabajos de Ana María Cousillas (1997); Mirta Bialogorski y Ana María Cousillas (2000; 2003); Mirta Bialogorski y Paola Fritz (2012).
No obstante, destacamos el pionero trabajo realizado a partir de la década del noventa en el Museo de Arte Popular "José Hernández" (Buenos Aires). ${ }^{32}$ En este caso, entre las medidas más eficaces, se cuentan la modificación de la imagen institucional y la reformulación del guión museográfico; estas fueron tomadas al detectarse que los visitantes tendían a interpretar el patrimonio del establecimiento asociado a lo gauchesco, lo telúrico, lo del interior. ${ }^{33}$

Los sucesos y transformaciones que se fueron dando en aquellas décadas en los museos argentinos en general $-y$ de arte en particular-, veinte años después, se encuentran internalizados en muchas de las dinámicas del hacer museístico cotidiano. Esta entidad patrimonial en la contemporaneidad se ofrece de manera fragmentada, diseminado en una diversidad de opciones de tiempo y de espacio; un gran número de ofertas masivas e individuales establecen una amplia diversidad de consumo y participación para distintos visitantes que se acercan a disfrutar de ellas. Ahora bien, ¿̇quiénes son los visitantes de los museos en Argentina?

El informe de la Encuesta de Consumos Culturales y Entorno Digital, Teatro y otros consumos culturales, generado en 2014 , brinda algunos datos que permiten delinear someramente los perfiles de los visitantes que consumen el museo como una actividad cultural. En líneas generales, no señala la presencia de diferencias significativas entre varones y mujeres en cuanto a la asistencia. Sí sucede, por el contrario, que se marquen distinciones según la franja etaria de los visitantes; allí se indica, por ejemplo, que las personas mayores de sesenta y cinco años asisten con menos frecuencia que las menores de cincuenta. Al acompañar estos datos con los aportados por la actualización de 2017, puede observarse que casi un tercio de la población asistente pertenece a los estratos socioeconómicos más altos. Además, se desprende también de la concurrencia que esta actividad es mayormente elegida por la población con nivel educativo terciario, universitario y postuniversitario.

Más allá de los variados alcances de estos datos, los que aquí seleccionamos presentan, desde nuestra perspectiva, una particular relevancia. No obstante, los criterios que se utilizan en el Informe no ofrecen materia suficiente para generar definiciones referidas a los rasgos de los visitantes de los museos de arte del siglo XXI. De hecho, estos análisis, a su vez, ayudan a reflexionar sobre qué datos está priorizando la políitica de Estado y cuáles son los alcances efectivos de un registro de datos basado en criterios estadísticos socio-demográficos. Nos preguntamos, por ejemplo, hasta qué punto interesa identificar el sexo de los asistentes, en la medida en que prima la búsqueda de diversidad; y cuán efectistas y diferenciadoras pueden ser las políticas institucionales en este orden. Sin embargo, se trata de interrogantes que dejamos abiertos para otro tipo de análisis. 
El segmento de museos escogidos, aquellos que se crearon en los últimos años, cobra un atractivo singular asociado no solo con la presentación de lo artístico, sino también con sus ofertas de espacios-servicios recreativos y de consumo. Se trata de un modelo que no escapa a la nueva dinámica que van adquiriendo las sedes museales en la actualidad en relación con su condición acendrada de instrumentos del sistema capitalista. Tal como adelantamos, abordaremos este fenómeno de los visitantes de los museos de arte argentinos desde las peculiaridades contextuales y las políticas institucionales de un caso en particular. Para ello, nos valemos del uso de un estudio de visitantes que combina los tipos de datos — tanto del orden cualitativo como cuantitativo - y busca atender a una variedad de imaginarios, necesidades, perfiles e intereses. Buscamos, de este modo, intentar posibles respuestas a nuestros interrogantes y, a su vez, comenzar a trazar líneas o registros tentativos que permitan construir indicadores generales.

\section{LOS VISITANTES DEL MUSEO DE ARTE DE TIGRE (MAT)}

La ciudad de Tigre, ubicada apenas a treinta kilómetros de la Capital Federal, supo ser, en las primeras décadas del siglo XX, el destino predilecto de la aristocracia porteña, ya sea para vacacionar o pasar los fines de semanas. En la década del ochenta, esta urbe alcanzó un nuevo apogeo al convertirse en parque industrial, activación que, además, fue acompañada por la expansión del servicio ferroviario y la explotación comercial de los inmuebles colaterales situados en una de las zonas más privilegiadas del conurbano bonaerense- - Su puesta en valor, durante los años noventa, la convirtió en símbolo de una época marcada por la exacerbación del consumo y la llegada de una nueva oleada turística; dejó de ser una zona marginal para volver a tener el esplendor de épocas pasadas. Bajo una estructura que vinculaba lo público con lo privado, se crearon el tren turístico de la Costa (Retiro-Delta, 1995), el Parque de la Costa (1997), el Casino Flotante (1999) y locales de primeras marcas, junto con restaurantes y cines; todos ellos acompañaban al conocido centro comercial de productos artesanales, el Mercado de Frutos. Una década más tarde, este proyecto de crecimiento urbano comenzó a mostrar signos de decadencia en concordancia con la situación económica nacional. Sin embargo, el municipio y parte del sector privado continuaron sosteniendo algunas de las propuestas recreativas y de esparcimiento, dirigidas a solventar la actividad turística, con el propósito de ampliar la oferta y atraer al turismo extranjero. 


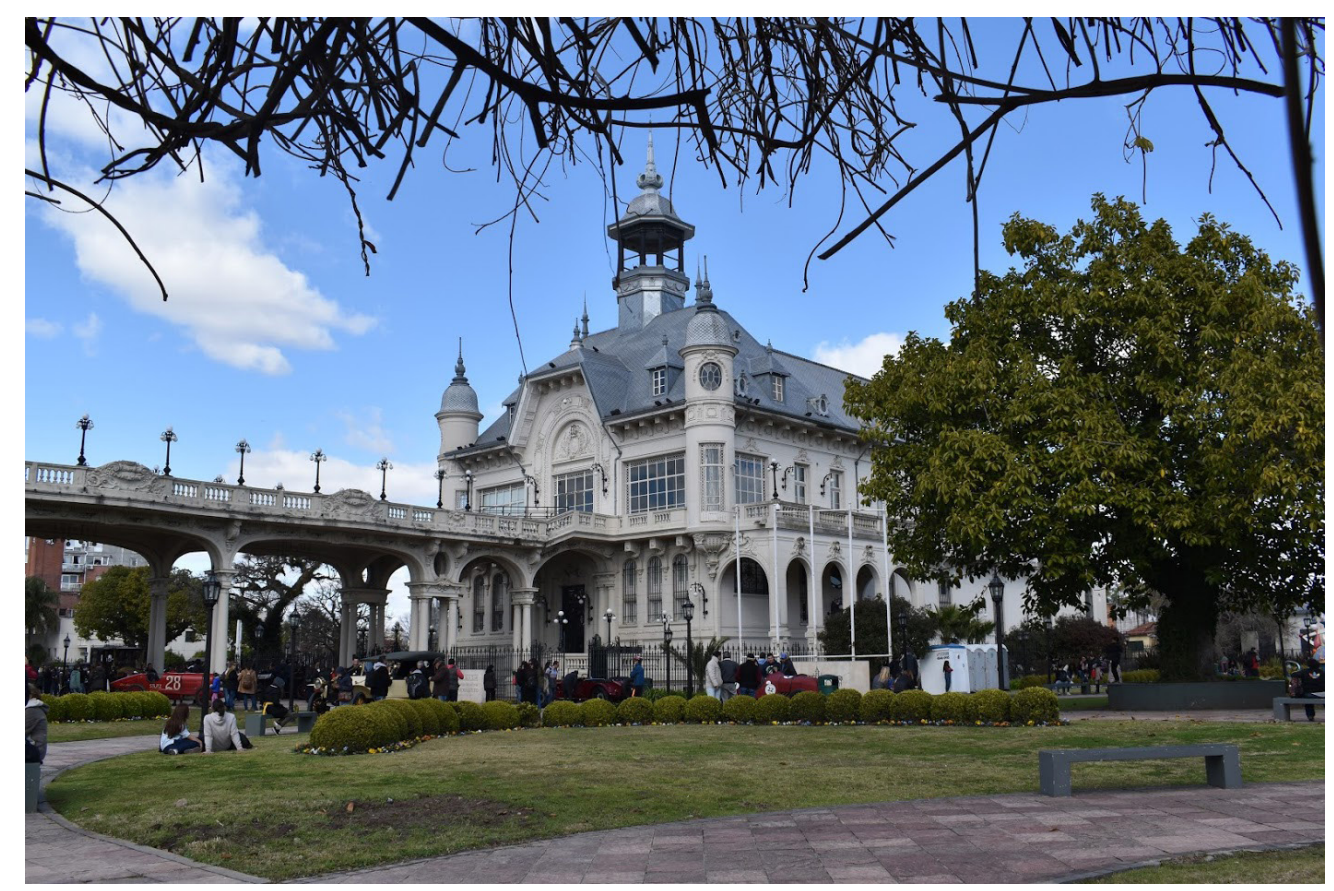

Figura 1 - Vista externa del Museo de Arte de Tigre (MAT). Créditos de imagen: Municipio de Tigre; Graciela Arbolave (Directora); Julieta Sosa (Prensa).

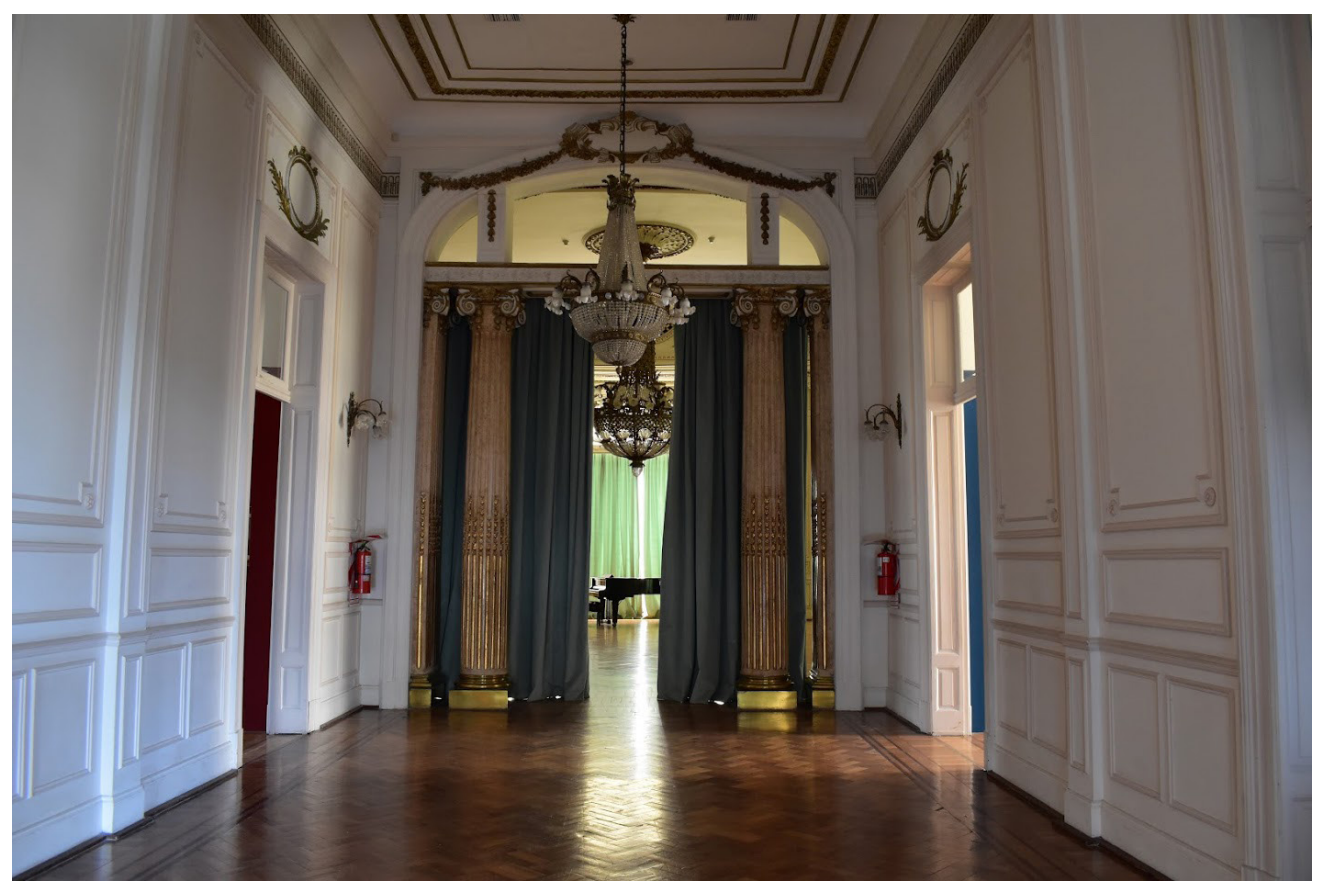

Figura 2 - Vista interna del Museo de Arte de Tigre (MAT). Créditos de imagen: Municipio de Tigre; Graciela Arbolave (Directora); Julieta Sosa (Prensa). 
En sintonía con esta lógica, en 1998, se inició la restauración del edificio del Tigre Club (1912), que posteriormente daría alberge al Museo de Arte de Tigre (MAT); este último, no obstante -y debido, en parte, a la crisis económica que había atravesado el país -, recién abrió sus puertas en 2006. La entidad patrimonial, ubicada en el casco histórico de la ciudad, se encuentra al final del paseo Victorica. Se trata de un edificio de dos plantas, de principios del siglo XX, con preponderante arquitectura neoclásica y rodeado de un amplio espacio verde. La planta baja del edificio cuenta con dos grandes salas centrales y otras más pequeñas ${ }^{34}$ que se comunican, por medio de una amplia escalera, con el primer piso. Este posee una sala expositiva y de reunión de importantes dimensiones antiguo salón de baile - con pequeños recintos a sus lados que culminan, en el otro extremo, en una pasarela con doble columnata y terraza a orillas del río Lujan. ${ }^{35}$ El museo brinda, tanto en su interior como en su exterior, no solo exposiciones con visitas guiadas, sino también talleres y cursos, actividades lúdicas y educativas, encuentros, conciertos y eventos masivos (figuras 1 y 2 ).

En cuanto a la colección del MAT, esta fue iniciada por el intendente Ricardo Ubierto ${ }^{36}$ y posee, actualmente, más de doscientas obras. La mayoría de ellas adquiridas por medio de remates públicos con presupuesto estatal destinado a ese fin - recorren el arte argentino figurativo de los siglos XIX y XX, con abordajes de distintos géneros, como el paisaje, el retrato o las costumbres del país. Entre sus piezas destacan las de artistas nacionales como Antonio Berni, Fernando Fader, Raúl Soldi, María Obligado, Santiago Cogorno y Norah Borges. El acta fundacional del museo explicitaba que la colección se debía exponer de manera permanente bajo los lineamientos del arte figurativo de los siglos mencionados. Sin embargo, a partir de la gestión de Diana Saiegh (2008-2012), se reconfiguró la programación anual; el objetivo era incorporar otras obras y abrir nuevos horizontes para ampliar los públicos. En este sentido, se ofrecieron muestras temporarias de artistas convocantes o productores contemporáneos y se desplegó un mayor número de actividades educativas; también, se organizaron encuentros sociales de privados, tales como desfiles, presentaciones y audiovisuales. Durante la gestión de María José Herrera (2014-2018) se continuó con algunos puntos de esta dinámica, pero con vistas a expandir el alcance de estas transformaciones. Su intención era dar mayor visibilidad al MAT en todo el país; para ello recurrió a muestras que promovían la difusión del arte argentino de manera accesible y didáctica para públicos diversos. En sus palabras: "Pretendo hacer grandes exposiciones para que entiendan un poco más sobre el mundo que nos rodea, que mostremos el arte contemporáneo. Que el museo sea un lugar de encuentro para toda la familia. Cruzar turismo con cultura es una muy buena opción". ${ }^{37}$ Otro rasgo que
34. En la parte inferior, también funcionan una pequeña tienda, y tanto en el piso inferior como en el primero, se ubican varias salas de personal.

35. Todo el edifico cuenta con un gran número de escaleras, por lo que se refaccionó un ascensor de la época y se agregó una silla eléctrica para permitir un mejor acceso a personas con dificultades motrices.

36. Elegido en primer mandato durante el gobierno de facto $1979-1983$ y reelegido, posteriormente, durante en cuatro oportunidades 19911995-1999-2003-2007.

37. Municipio de Tigre (2014). 
38. La colección que Simón Scheimberg, donada al Museo Nacional de Bellas Artes, a comienzos de los años setenta, cuenta con obras de Luis Seoane, José Laxeiro, José Palmeiro, Manuel Colmeiro; artistas de la Escuela de París, Bigatti, Forner, Spilimbergo, Pettoruti, Gómez Cornet, Guttero y Basaldua, Lacámera, Victorica, Del Prete, Daneri, Diomede. Junto a ellos Martín Malharro y Eduardo Sívori, Domingo Viau, Juan Carlos Castagnino, Enrique Policastro, Ramón Silva, Georges Braque, Henri Matisse, Pablo Picasso, Auguste Rodin, Kathe Kollwitz, Georges Rouault, Theophile Steinlen, Louis Forain, Cándido Portinari, Henri de Toulouse Lautrec, entre otros.

39. Las exposiciones eran acompañadas por dos visitas guiadas programadas respectivamente a las $15.00 \mathrm{y}$ las 17.00 horas.

40. Se trata de un proyecto generado por la Universidad Nacional de Tres de Febrero (UNTREF) que brindó exhibiciones artísticas en ochenta y cuatro sedes de treinta y dos ciudades, en dieciséis países.

41. La obra simula un xilofón desplegado en un espacio de sesenta metros que al ser ejecutado va revelando un fragmento de la novena sinfonía de Beethoven.

42. La pieza presenta un péndulo de madera que al final de cada ciclo del movimiento pendular se encuentra una copa con agua que, al ser tocada sutilmente, parece a punto de derramar el líquido.

43. Dato suministrado por personal del museo a partir del relevamiento de entradas.

44. Las entrevistas se realizaron entre el 05/08/2017 y el 24/09/2017; la mues- conviene destacar es la atención dedicada a la relación entre educación y entretenimiento, que se expresa en ese cruce entre turismo y cultura.

Durante los meses de agosto y septiembre de 2017, el MAT ofreció en sus salas superiores una exposición que se basó en una investigación interdisciplinaria referida a la Belle Époque de la ciudad de Tigre; esta abarcaba desde 1880 a la Primera Guerra Mundial, e incluía la construcción del Tigre Club. El fin de esta muestra era recuperar la historia del lugar que, hasta entonces, había estado ausente de las salas de la sede museal. Asimismo, en ese piso se mostraba una de las líneas más potentes de la colección, los paisajes; una orientación que hacia 1910 se consideraba una de las temáticas nacionales por excelencia. Simultáneamente, en las salas inferiores se ofrecían tres exposiciones temporales con piezas de lenguajes tradicionales. La primera, llamada Ernesto Deira, los fragmentos del conflicto reunían un conjunto de dibujos de uno de los pintores de la Nueva Figuración argentina. Las otras dos exposiciones se concentraban en la importancia de los coleccionistas en la historia del arte argentino; por un lado, se mostraban piezas de la colección del abogado Simón Scheimberg, bajo el título Colección Scheimberg: arte para el pueblo ${ }^{38}$ y, por otro, de la colección del Dr. Mauricio Neumann, con el título Antonio Berni en la colección Neumann. Dichas exhibiciones buscaban evidenciar un modelo no tradicional de coleccionismo que se entroncaba con momentos claves del arte y la políitica en el siglo XX, y permitían dilucidar las elecciones atravesadas tanto por sus afinidades estéticas como por las convicciones ideológicas de estos actores. ${ }^{39}$

Durante estos meses, la programación contó también con algunos eventos especiales. Durante el mes de septiembre, el MAT fue una de las sedes de la primera Bienal de Arte Contemporáneo de América del Sur (BIENALSUR). ${ }^{40}$ En su parque se exhibió "Corre por la música", de Leandro Erlich, ${ }^{41}$ y en una de las salas superiores, "4,33 metros", de Pablo Nenflidi. ${ }^{42}$ Al mismo tiempo, se ofrecieron distintos eventos de música de cámara y jazz; talleres de plástica para niños; reuniones de artistas que formaron parte de la Bienal; la fiesta de autos clásicos; entre otras actividades generales.

Entre agosto y septiembre de 2017, la asistencia durante los fines de semana al MAT alcanzó, aproximadamente, las 3000 personas. ${ }^{43}$ Pero żquiénes fueron estos visitantes? Para intentar una respuesta, decidimos llevar adelante, durante los meses señalados, un estudio que se concentró en las diferencias y las singularidades de los micropúblicos dentro del conjunto mayor del público de fin de semana -el menos caracterizado en este tipo de entidad patrimonial - . El trabajo de campo tuvo lugar durante estos meses, un día del fin de semana, en los horarios de apertura y cierre del establecimiento. ${ }^{44}$ La recolección de los datos se instrumentó mediante breves entrevistas semiestructuradas que llevé adelante personalmente; se efectuaron en español, aunque también fueron respondidas por hablantes de otras lenguas - 
portugués, francés, inglés - que manifestaban comprender el idioma. Se realizaron en la puerta del museo a todos aquellos visitantes que se mostraron dispuestos tras finalizar su recorrido; la tarea dio por resultado un total de 383 personas consultadas, de diversos rangos etarios y nacionalidad. La entrevista consistía en dieciséis preguntas; cinco de carácter sociodemográfico y el resto sobre la experiencia de la visita al museo - algunas con presentación de opciones, y otras, abiertas-.

Para comenzar con nuestro análisis, primero nos referiremos a aquellos datos que conceden una construcción de orden cuantitativo y más general sobre los visitantes de este museo de arte. En este sentido, pudimos relevar que, durante los fines de semana, el 72\% de la presencia en el establecimiento correspondía a personas que visitaban ese museo por primera vez -también nombradas como público potencial ${ }^{45}$. . Cabe señalar que el alto porcentaje de asistentes que se referían a esa como su primera visita puede asociarse con dos factores ya mencionados: la locación del museo en un punto turístio, y el hecho de ser una joven institución con apenas doce años de antigüedad. Solo un 23\% estaba conformado por individuos que lo habían visitado en más de una ocasión; entre estos últimos, la cantidad de visitas y los lapsos entre ellas presentaban un tipo de variación no nos posibilita considerarlos como un público asiduo. A su vez, estos datos permiten reconocer que, mayoritariamente, los visitantes del fin de semana configuran un público potencial. ${ }^{46} \mathrm{Se}$ trata, entonces, de manera preponderante, de individuos que visitan el MAT de forma circunstancial: "vinimos a pasar nuestro aniversario y estábamos paseando por la costa y vimos el museo"; "nos recomendaron la muestra de Berni"; "estábamos en el parque merendando y decidimos conocer el museo que está al lado"; "toca un amigo en el concierto y vamos a escucharlo"; "nos tocó este museo para completar la tarea que le dio la señorita al nene"; "estamos recorriendo los espacios donde se pueden ver las obras de la Bienal de Arte".

Ahora bien, ¿qué más podemos saber acerca de este público? Otro aspecto pasible de profundizarse es su perfil sociodemográfico. Prima la asistencia de adultos entre 36 y 65 años - el 44\% - y con un alto nivel de estudios universitarios - el 53\%-; estas condiciones se presentan en continuidad con los datos aportados por la Encuesta de Consumos Culturales en Argentina. Se trata de un aspecto que nos permite recuperar algunas ideas de los estudios de público históricos ${ }^{47}$ realizados en los museos de arte. Según dichos estudios, la visita a tales museos se asociaba, principalmente, a un conjunto de individuos que gozaban de cierto nivel educativo y que pertenecían a una clase social determinada, lo que conllevaba que esta actividad fuera considerada, justamente, un ritual de clase. Esto nos lleva a preguntarnos sobre la efectividad de los intentos tra resultante se corresponde, aproximadamente, con un $10 \%$ del total de visitantes de los fines de semana de agosto y septiembre de 2017.

45. Esta posición se sostiene en línea de indagación propuesta por Luciana Sepúlveda Köptcke (2012).

46. Ibid.

47. Cf. Bourdieu; Darbel (2002). 
48. Aquí, nos referimos a los textos de Andrea Fraser (2007), Ana María Guasch (2008) y Andreas Huyssen (2010) actuales por democratizar esta institución cultural. Resulta insoslayable que la experiencia que proporcionan los museos de arte continúa siendo escogida solo por algunos individuos con características determinadas. Y cabe interrogarse, asimismo, acerca de cuánto de ello se debe, por ejemplo, a la falta de políticas estatales en los sectores cultural o educativo; o a la carencia de algunos proyectos impulsados por distintas gestiones para vincular lo artístico, lo museístico y la vida cotidiana. Por último, algunos indicios parecen sugerir, según lo han hecho notar varios autores, ${ }^{48}$ la posibilidad de una restructuración de las clases sociales, producto de las últimas transformaciones del capitalismo global.

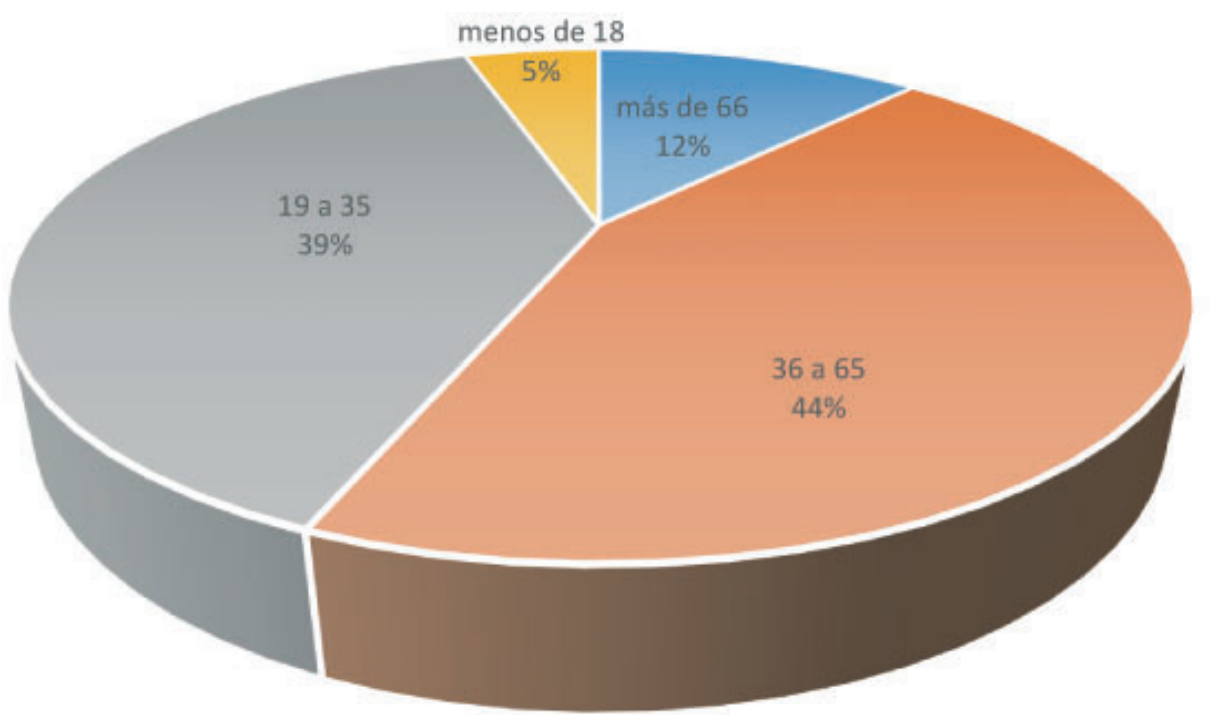

Gráfico 1. Rango etario de los visitantes entrevistados.

Al indagar el lugar de procedencia de los visitantes, se registró que solo un $12 \%$ de ellos formaba parte de la comunidad de Tigre, mientras que la mayoría -el $88 \%$ - no se encontraba radicada allí - un $63 \%$ de personas provenían del Gran Buenos Aires; un 19\% estaba compuesto por extranieros; y el restante $6 \%$ lo integraban individuos de otras provincias argentinas - . Este último dato puede vincularse con las características propias de la ciudad, ya que recibe una gran afluencia de visitantes, pues se trata de una zona turística. La sede museal, por otra parte - como ya dijimos - , se encuentra ubicada en el paseo ribereño, entre las otras ofertas de entretenimiento del circuito turístico. Esta situación ya fue señalada cuando se observó que la mayoría de los entrevistados manifestaban visitar ese museo por primera vez. 


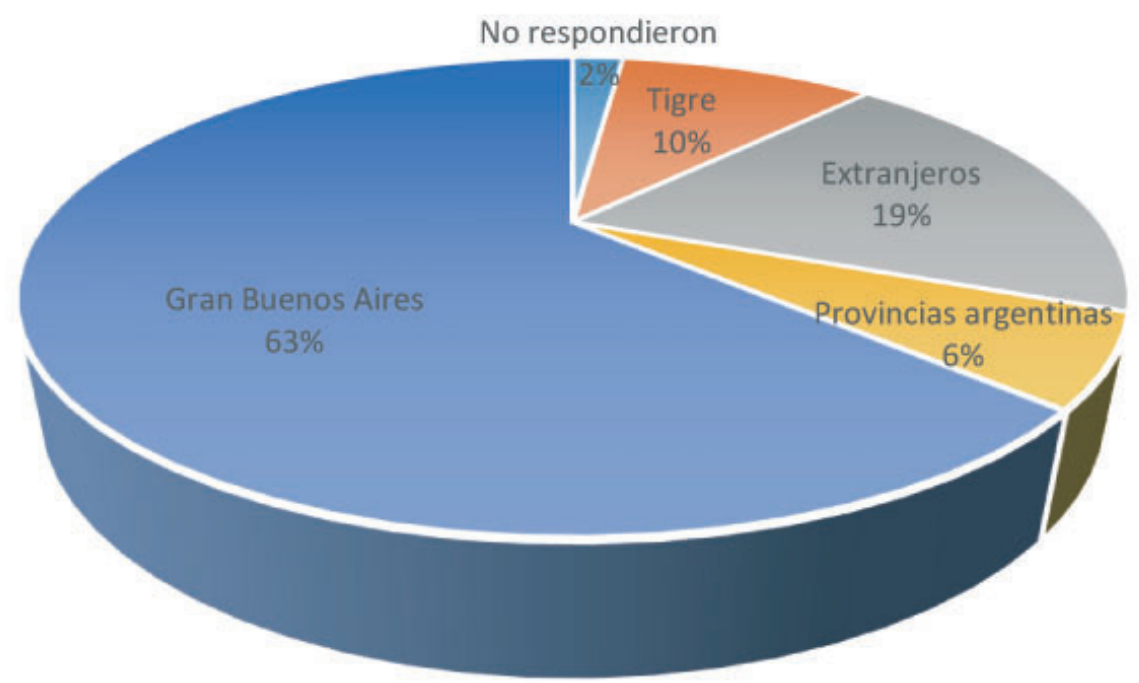

49. Piacentino (2009, p. 29).

Gráfico 2. Lugar de procedencia de los visitantes entrevistados

El conjunto de estos datos posibilita distinguir algunos rasgos de los visitantes actuales que se conectan con el perfil adoptado por la entidad patrimonial a partir de 2012. En este marco, el MAT parece orientar su interés hacia los visitantes ocasionales y turistas, con una programación que busca educar y entretener en línea con la dinámica adquirida por este tipo de institución cultural en el siglo XXI. No obstante, la colección y las exposiciones temporales apuntan marcadamente a privilegiar y destacar aspectos del arte argentino. Esta manera de presentación, brinda a los visitantes ocasionales extranjeros información particular sobre este tipo de arte, pero también atiende a las necesidades de los turistas nacionales o cercanos con relecturas sobre lo nacional y lo local. Todo ello se coordina con actividades culturales u ofertas de entretenimiento más generales. Esta dinámica puede ser percibida como un intento de generar una entidad flexible que, a su vez, no desdibuja las funciones que la caracterizan; todo ello, con el fin de ser recomendada a otros o escogida nuevamente. La contracara de esta lógica es que algunos de los vecinos de Tigre consideran que las ofertas recreativas con privados o el privilegio de exposiciones temporales sobre la colección "han desvirtuado absolutamente el espacio, la finalidad de la institución y el destino del patrimonio del museo"; 49 quizás esta lectura pueda proporcionar una primera pista en relación con la escasa concurrencia de este tipo de público. 
50. Cf. Pekarik, Schreiber, Hanemann, Richmond y Mogel (2014).
En otro orden, dado que nos enfocamos en los visitantes de los fines de semana, en cuanto a los contextos en que se encuentran dichos visitantes, se acentúa, particularmente, la presencia de público familiar - un 62\% del total, conformado por $31 \%$ de familias con diferentes estructuras y edades, y un $31 \%$ de parejas-. Quedan, por detrás, las visitas con amigos - el 28\% - las de individuos que asisten solos — un $8 \%$ - y las de grupos integrados por profesionales - que constituyen el $4 \%$ - Estamos ante un conjunto de visitantes que busca con claridad una experiencia compartida, social y alejada de la cotidianidad. En este sentido, es sustancial distinguir las ofertas de la ciudad y del MAT para asistir a estos tipos de visitantes. Esta zona de Tigre, que se extiende a lo largo de la costa, brinda la conjunción del espacio verde con propuestas recreativas y de consumo que se complementan, a su vez, con la invitación a visitar la sede museal. Esta entidad brinda, durante los fines de semana, talleres de plástica para niños, visitas guiadas y una variada oferta expositiva, a manera de amalgama entre el entretenimiento y lo educativo. Ambas condiciones, podemos considerar que concentran las preferencias de estos micropúblicos; ofertas que permitan divertirse y enriquecerse con la transmisión de conocimiento.

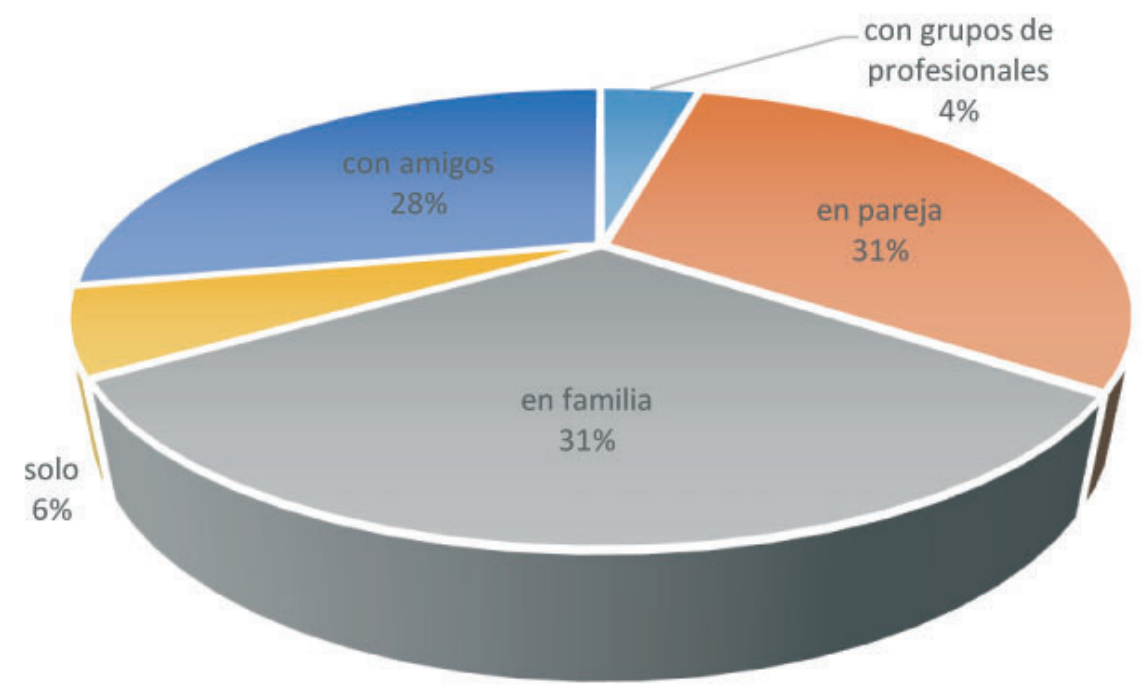

Gráfico 3. ¿Con quién visitaron el MAT?

Si nos detenemos, ahora, en la tipología del museo, podemos profundizar en un análisis de orden cualitativo en relación con las singularidades propias de los visitantes de un museo de arte. ¿Qué preferencias tienen quienes asisten a estas sedes? Las categorías de las preferencias en relación con la experiencia de la visita ${ }^{50}$ pueden brindarnos algunos indicios; su lectura requiere cierta flexibilidad, en vista del marcado carácter personal e idiosincrásico de algunas respuestas obtenidas. 
En primera instancia, reconocemos un alto número de expresiones que se vinculan con la lectura de los objetos; nos referimos a aquellos individuos que 52. Ibid. marcaron un interés por obras particulares. Esta perspectiva, puede leerse, por ejemplo, en expresiones como: "son impresionantes algunos de los cuadros"; "me fascinaron las pinturas con los rostros en negro de Deira"; o "entramos al salón oval para ver el cuadro de la colección, el de Quinquela". Se percibe que el visitante, aunque llegue por distintas razones, sabe con qué se va a encontrar allí; es decir, con un objeto museístico que atiende a su condición de obra de arte, y en el que se privilegia el valor simbólico por sobre el valor de uso, ${ }^{51}$ en pos de establecer una experiencia estética - incluso es posible que se le atribuya cierto grado de sacralidad - . Sin embargo, al profundizar nuestro análisis, se manifestó, además, la existencia de otros dos públicos, uno explícitamente aficionado al arte y otro caracterizado por su juventud; ambos se diferenciaban del conjunto anterior por la búsqueda o la demanda de un mayor número de propuestas plásticas contemporáneas - recordemos que en el MAT solo se presentaron, durante el mes de septiembre, dos producciones contemporáneas, producto de BIENALSUR - . Tanto en el público experto como en el joven primaba una valoración de las obras contemporáneas como capaces de generar otra interacción, no limitada meramente a la contemplación durante el recorrido. Tal vez, esta interacción con propuestas contemporáneas se pueda asociar con la categoría de preferencia física, ${ }^{52}$ es decir, un interés porque el recorrido incluya experiencias sensoriales o de movimiento ligadas a recursos audiovisuales o a piezas multimedia, instalaciones, intervenciones, etc., y no orientadas solo a lo contemplativo. En síntesis, el público general - al que podríamos caracterizar como adulto y no especializado en arte- mostraba - a diferencia de lo que ocurría con los expertos y los jóvenes - una clara preferencia por obras de lenguajes, materialidades y formatos más clásicos, tales como los ofrecidos en las exposiciones señaladas.

Es de notar que, en todos los casos, se observó un reconocimiento sustancial de la figura del artista; era importante para la gran mayoría de los visitantes saber quién era el autor de la obra en su contacto con ella. En este sentido, se puede especular acerca del motivo de que una de las muestras temporales presentase obras de Antonio Berni, quien se cuenta entre los íconos de la producción plástica de Argentina. Las materialidades y las temáticas de sus reconocidos "Juanitos" y "Ramonas" - piezas emblemáticas en la obra de este artista de prestigio nacional e internacional - suelen atraen a un gran número de fieles dentro y fuera del país. Esta referencia habilita a pensar en la incorporación del tipo de muestras denominadas blockbusters a la programación del MAT. Se trata de una estrategia para capturar a un mayor número de visitantes que, así como puede apelar al abordaje de temas 
53. Ibid.

54. Cf. Sepúlveda Köptcke (2012). populares, también puede recurrir a figuras convocantes del mundo de las artes como parte de un repertorio que garantiza cierta masividad y visibilidad. Estos aspectos, tal como mencionamos, están en línea con las lógicas curatoriales de los museos en la contemporaneidad y con las decisiones de las gestiones del MAT desde 2012.

Otro aspecto que se desprende de lo que venimos comentando acerca de las preferencias de los visitantes de estos museos es su elección con respecto al programa de exposiciones. Se aprecia, en este sentido, un interés por las razones del agrupamiento de las piezas, tanto de las muestras temporales como de la colección, en coincidencia con el beneplácito ante el abandono de la linealidad histórica en la narración; los visitantes se sienten interpelados de un modo estimulante por las relecturas sobre lo artístico en clave nacional y local. De allí expresiones tales como: "me quede impactado con las diferentes obras y autores argentinos que no conocía" o "es interesante como esta armada la muestra, me gustaron los núcleos de los paisajes y las naturalezas muertas". Esta condición se podría vincular con una preferencia por las ideas, pero, a nuestro entender, no llega a destacarse tan fuertemente como para considerarla por sí sola en el sentido que plantea; ${ }^{53}$ pareciera, en cambio, estar más determinada por la preferencia vinculada a la lectura de los objetos.

En segunda instancia, es sustancial, entre las preferencias mencionadas por los visitantes, el lugar que ocupa el interés por la interacción social. Específicamente, se destacaron dos grupos de expresiones. Por un lado, se cuentan las referidas a los espacios-servicios como aquellos que permiten disfrutar de vínculos de sociabilización: "es impresionante la vista que hay desde la terraza, estuvimos sentados charlando un largo rato apreciando la vista"; "ahora vamos a tomar unos mates en el parque"; o "estábamos disfrutando del parque; entramos, pero aún tenemos tiempo de disfrutar un rato más del verde". Por otro, se observan palabras de reconocimiento hacia el recorrido acompañado, ya que se generaba una interacción social a partir de las personas asignadas como guías: "nos encantó la guía" o "aprendimos un montón sobre el edificio y la obras con las explicaciones de la guía".

La posibilidad de determinar quiénes son los visitantes de estas jóvenes entidades argentinas enfocadas en el arte se encuentra en un momento de desarrollo aún incipiente, pero sí podemos brindar algunas aproximaciones sobre el perfil de los visitantes que recorren, durante los fines de semana, el MAT. En líneas generales, sobresalen tres tipos de público. En primer lugar, un público potencial, en el sentido de grupos que poseen características socioculturales similares a las de los micropúblicos asiduos, y que visitan instituciones culturales similares en otros lugares; a ello se suma que ya hicieron una primera visita. En virtud de todos estos rasgos, pueden llegar a convertirse en visitantes o públicos de este recinto. ${ }^{54}$ Los otros dos 
tipos de público que se destacan son el turístico y el familiar, que no se excluyen mutuamente; respecto de estos dos tipos, deben considerarse, no solo el carácter turístico de la locación y el factor de que las entrevistas se realizaron durante el fin de semana, sino también las particularidades propias de los museos en la contemporaneidad - en especial, lo relativo a los espacios-servicios, las zonas verdes, los servicios, atracciones comerciales-. En cuanto a su tipología específica, se observa la presencia de individuos interesados en una lectura de los objetos - la obra de arte- y por la interacción social - espacios de sociabilización con otros - Todos ellos muestran tener preferencia por un tipo de institución cultural, aquí representada por el MAT, que no solo dé lugar a instancias educativas sino que también habilite lo recreativo. Este cruce, permite leer "una reconciliación entre musas y masas". 55 En este sentido, podemos vincular el perfil que este museo fue adquiriendo en los últimos años con el ofrecimiento de propuestas expositivaseducativas que intentan mediar entre lo formativo y el entretenimiento; se brindan, por ejemplo, relecturas sobre el arte argentino, en conexión con piezas de artistas destacados y se combinan con recursos o guías para su interpretación. Asimismo, esta orientación se complementa con espacios-servicios - espacio verde, terrazay eventos y actividades culturales que invitan a la recreación. Todo ello se corresponde fuertemente con las otras ofertas del circuito turístico de la ciudad.

Esta conjunción de factores, acorde con aquello que debe hacer y ofrecer un museo de arte en la contemporaneidad, permite reconocer al MAT como una de las sedes museales más visitadas por fuera del circuito porteño. No obstante, en esta incipiente indagación, se detecta que cada uno de los subgrupos de públicos señalados presenta diferentes necesidades y búsquedas por ser atendidas; pero, a la vez, se deja al descubierto la existencia de otros visitantes que están siendo descuidados o no atendidos. Los públicos de los museos presentan un rasgo de complejidad asociado con la multiplicidad de regímenes espectatoriales y de consumo, lo cual pone de relieve, simultáneamente, cuán complejo resulta atenderlos ante lo variado y fluido de su composición.

\section{COMENTARIOS FINALES}

Aún queda mucho camino por recorrer para acercar respuestas más certeras sobre quiénes son los públicos de los museos de arte. Este trayecto ofrece un posible inicio; quiere pensarse, ante todo, como un conjunto de pistas que permitan poner el foco en -y atender a- otros visitantes. Dicha atención hará posible construir 
56. Cf. Panozzo Zenere (2018). opciones diferentes de aquellas consideradas clásicas, las cuales tradicionalmente marcaron la construcción del perfil de un público específico de estas sedes museales. Es necesario comprender que el museo de arte en la contemporaneidad recibe una multiplicidad de individuos que se acercan a conocerlos. En tal sentido, resulta insuficiente aplicar patrones que solo atienden a fenómenos de regularidad; por el contrario, consideramos oportuno comenzar a desagregar los conjuntos y registrar las subdivisiones presentes entre sus visitantes.

El caso de análisis, a pesar de su marco restringido en cuanto al tiempo disponible y el tamaño de la muestra, ofrece una exploración para comenzar a pensar de manera plural en los visitantes de los museos de arte creados a partir del siglo XXI. Ello abre, asimismo, la posibilidad de idear estrategias para fidelizarlos, en cambio de apelar solamente a eventos particulares dentro de la programación o a la oferta de servicios, espacios y actividades realizada sin que se tenga en claro la tarea que se busca llevar adelante.

Los museos de arte argentinos, al igual que muchos otros, se encuentran frente a un reto. No se trata únicamente de aumentar el número de visitantes siguiendo la lógica del sistema capitalista - , sino de atraerlos y de atender a una mayor diversidad de ellos con el objetivo principal de combatir la desigualdad en el acceso a la cultura. Al respecto, se ven en la necesidad de interrogarse, una vez más, sobre su rol en la actualidad; en particular, si se tiene en cuenta - como hemos dejado entrever aquí y propusimos en otro trabajo ${ }^{56}$, su papel disciplinador.

Las respuestas no son claras ni unívocas, y hasta podemos decir que, en algunos casos - en relación con las políticas culturales estatales y las gestiones-, cobran un carácter muy singular. Sin embargo, nos gustaría pensar que insistir en los interrogantes puede contribuir a impulsar búsquedas más plurales y democráticas, y a generar diálogos en torno a una construcción de sentidos que respondan a los diversos intereses de todos los actores involucrados. 


\section{Referencias}

LIBROS, ARTÍCULOS Y TESIS

ALDEROQUI, Silvia; PEDERSOLI, Constanza. La educación en los museos: de los objetos a los visitantes. Bs. As: Ediciones Paidós, 2011.

ANDERSON, Chris. La economía log tail. México: Empresa activa, 2009.

BALLO, Guido. Occhio Critico. Il nuovo sistema per vedere l'arte. Milano: Longanesi, 1966.

BENNETT, Tony. Exhibition, difference, and the Loginc of Culture. In: Karp I. (Comp.). Museum Frictions. Nueva York: Duke Univesity Press, 2007, p. 46-69.

BENNETT, Tony. The exhibitionary complex. In: Greenberg, G. (Ed.). Thinking about Exhibitions. London: Routledge, 1996, p. 69-92.

BIALOGOSKI, Mirta; COUSILLAS, Ana María. Gestión cultural y estudios de Público en el Museo José Hernández. Cuadernos de Antropología Social, Buenos Aires, n. 12, p. 197-205, 2000.

BIALOGOSKI, Mirta; COUSILLAS, Ana María. Estereotipos y preconceptos en la interpretación del patrimonio cultural en el Museo José Hernández. Buenos Aires: Ediciones del Instituto Nacional del Arte (IUNA), 2003.

BIAlOGORKI, Mirta; FRITZ, Paola. Dialogo con los visitantes. Estudio de Publico cualitativos en el Museo de Arte Popular José Hernández de la Ciudad de Buenos Aires. Actas del Simposio Internacional de Museología, Nuevas Prácticas, Nuevas audiencias. A 40 años de la Mesa de Santiago, Santiago de Chile, 2012.

BITGOOD, Stephen. Environmental psychology in museums, zoos, and other exhibition centers. In: BECHTEL, Robert B.; CHURCHMAN, Arza (Eds.). Handbook of environmental psychology. New York: Willey, 2002, p. 46-480.

BOURDIEU, Pierre; DARBEL, Alain. El amor al arte. Los museos europeos y su público. Buenos Aires: Paidos, 2002.

BUTTLER, Barbara; SUSSMAN, Marvin. Museum Visits and Activities for Family Life Enrichment. Londres: Routledge, 1989. 
CHIRIGUINI, María Cristina. Del colonialismo a la globalización. In: CHIRIGUINI, María Cristina; GRAVANO, Ariel. (Comp.). Apertura a la antropología. Buenos Aires: Proyecto Editorial, 2008, p. 31-56.

COELHO, José Teixeira. Diccionario crítico de política cultural: cultura e imaginario, Guadalajara. Jalisco: CONACULTA, Secretaría de Cultura del Gobierno de Jalisco, 2000.

COUSILlAS, Ana María. Guía sobre estudios de visitantes a museos. Buenos Aires: INAPP, 1997.

FALK, John. Identity and the Museum Visitor Experience. California: Left Coast Press, 2009.

FALK, John; DIERKING, Lynn. The Museum Experience Revisited. California: Left Coast Press, 2013.

FRASER, Andrea. “¿No es un lugar maravilloso? (El tour de un tour por el Guggenheim Bilbao). In: GUASCH, Anna Maria; ZULAIKA, Joseba (Coord.). Aprendiendo del Guggheim Bilbao. Barcelona: Akal, 2007, p. 39-62.

GARDNER, Howard. Frames of Mind: The Theory of Multiple Intelligences. New York: Basic Books, 1983.

GIUNTA, Andrea. Vanguardias y arte contemporáneo. Arte Argentino Contemporáneo. Rosario: Ediciones macro, 2004, p. 15-21.

GUASCH, Ana María. Los museos y lo museal. El paso de la modernidad a la era de lo Global. Revista CALLE 14, n. 2, p. 11-20, 2008.

HONEY, Peter; MUMFORD, Alan. Using Your Learning Styles. Maidenhead. Berkshire: Peter Honey, 1995.

HOOD, Marilyn G. Staying Away: Why People Choose Not to Visit Museums. [S.1.], Museum News, 61(4), p. 50-57, 1983.

HUYSSEN, Andreas. En busca del futuro perdido: Cultura y memoria en tiempo de globalización. Buenos Aires: Fondo de Cultura Económica, 2010.

KRAUSS, Rosalind. Arte en tránsito. La lógica cultural del museo tardocapitalista. $A V$ : Monografías, v. 39, p. 16-25, 1993. 
LASECA, Roc. El museo imparable: sobre institucionalidad genuina y blanda. Santiago de Chile: Metales Pesados, 2016.

LORD, Barry; DEXTER, Lord; MARTIN, Linday. Manual of Museum Planning: Sustainable Space, Facilities and Operations. UK: Altamira Press, 2012.

LORENTE LORENTE, Jesús Pedro. Museología Crítica y arte contemporáneo. Zaragoza: Prensas Universitarias Zaragoza, 2003.

MAIRESSE, François. El museo hibrido. Buenos Aires: Ariel, 2013.

OLIVERA, Elena. Cuestiones de arte contemporáneo. Hacia un nuevo espectador en el siglo XXI. Buenos Aires: EMECÉ, 2008.

ORR, Tori. The Information-Seeking Behavior of Museum Visitors: A Review of the Literature. 2004. Disponible en: <http://bit.ly/ROL_museumvisitors_TOrrTori>. Acceso en: 2 agosto 2018.

PANOZZO ZENERE, Alejandra. Se contempla, se experimenta. Modos de comunicar del museo de arte contemporáneo. Rosario: UNR Editora, 2018.

PEKARIK, Andrew; SCHREIBER, James; HANEMANN, Nadine; RICHMOND, Kelly; MOGEL, Barbara. IPOP: A theory of Experience Preference. Curator: The Museum Journal, California, n. 57, p. 5-27, 2014.

PÉREZ CASTELlANO, Leticia. Estudios sobre públicos y museos. Vol. I. Públicos y museos: ¿Qué hemos aprendido? Ciudad de México: Publicaciones ENCRy M, 2016.

PÉREZ CASTELLANO, Leticia. Estudios sobre públicos y museos. Vol. II. Apuntes para pasar de la teoría a la práctica. Ciudad de México: Publicaciones ENCRy M, 2017.

PIACENTINO, Ana. Gestión y políticas públicas en gobiernos locales. Caso: Museo de arte de Tigre, intendente Ricardo Ubierto. CESYT - Centro de Estudios Sociales y Tecnologicos, p. 41, 2009.

RUIZ BAÑON, Maria Luz. La imagen de los museos de arte contemporáneo: percepción del público visitante y no visitante. Madrid: Asociación Cultural y Científica Iberoamericana, 2017.

SEPÚLVEDA KÖPTCKE, Luciana. ¿Público, el X de la cuestión? La construcción de una agenda de investigación sobre los estudios de público en Brasil, v. 1, n. 1, p. 209-235, 2012. 
SHERMAN, Daniel; ROGOFF, Irit. Museum Culture: Histories, Discourses, Spectacles. London: Routledge, 2003.

SHOEMAKER, Maria. Watching children grow: a guide to childhood development. The docent educator, Philadelphia, v. 2, n. 1, p. 6-9, 1992.

VERÓN, Eliseo; LEVASSEUR, Martine. Ethnographie de l'exposition. L'espace, le corps et le sens. Paris: Bibliothe?que Publique d'Information - Centre Georges Pompidou, 1984.

SITIOS

MINISTERIO DE LA NACIÓN. Guía Nacional de Museos. Buenos Aires, 2013.

MORRIS HARGREAVES MCINTYRE. Never mind the width feel the quality. Museums and heritage show. London, 2005. Disponible en: <https://mhminsight.com/files/never-mind-thewidth-Tw57-68.pdf> . Acceso en: 25 abril 2018.

MUNICIPIO DE TIGRE. El MAT tiene una nueva Directora General. Secretaria de Tigre, Enero 15, 2014. Disponible en: <http://servicios.tigre.gov.ar/prensa/3708_el_mat_tiene_una_nueva_ directora_general_.htm>. Acceso en: 11 noviembre 2018.

Artículo presentado el 17/12/2018. Aprobado el 02/10/2019. (cc) BY 